Voix et Images

voixetimages

\title{
« Faire arriver ce qui n’existe pas encore »
}

\section{Claire Lejeune}

Volume 14, numéro 1 (39), automne 1988

France Théoret : narratrice de la subjectivité

URI : https://id.erudit.org/iderudit/200752ar

DOI : https://doi.org/10.7202/200752ar

Aller au sommaire du numéro

\section{Éditeur(s)}

Université du Québec à Montréal

ISSN

0318-9201 (imprimé)

1705-933X (numérique)

Découvrir la revue

Citer cet article

Lejeune, C. (1988). « Faire arriver ce qui n'existe pas encore ». Voix et Images, 14(1), 50-56. https://doi.org/10.7202/200752ar d'utilisation que vous pouvez consulter en ligne.

https://apropos.erudit.org/fr/usagers/politique-dutilisation/ 


\title{
«Faire arriver ce qui n'existe pas encore»
}

\author{
par Claire Lejeune, Université de Mons
}

Ma chère France,

J'écris, dis-tu, en pensant d̀ qui ne me lira jamais. Il n'y a pas de destinataires pour les aveux. Ta réponse à la question du destinataire me trouble. N'est-elle pas elle-même un aveu dont, te lisant, je suis malgré toi la destinataire? M'appropriant cette écriture qui se veut de pure perte, je m'en reconnais bénéficiaire. Il est indéniable que j'en ai fait et que je n'ai pas fíni d'en faire mon profit. À écriture de pure perte, écriture de pur profit? Toujours est-il que j'ai envie de t'en remercier, de partager le profit avec toi et de partager ce partage avec les lecteurs de cette lettre ouverte.

Ainsi, je voudrais faire de Entre raison et déraison une lecture contagieuse, qui soit un acte poétique; lecture interactive qui poursuive cette «correspondance à quatre voix» dont je décryptais les clefs dans une lettre à toi datée d'août 1985, à propos de «La turbulence intérieure». J'aimerais que cette lettre transporte notre dialogue en avant de ce qu'il fut jusqu'ici, que ton livre lui serve ainsi de tremplin; que ma lecture profite à nos livres à venir. En somme, faire une lecture-atelier, au sens où nous l'entendons, et qui devient génératrice d'écriture à partir du moment où le narcissisme primaire a été troué, dépassé; où la traversée du miroir nous donne accès a l'inconscient de tous, selon l'expression, citée par toi, de Louise Bouchard. C'est dans la pensée verticale - où la profondeur crée la hauteur - que le compagnonnage devient possible. L'essai poétique est propice au creusement de soi; à travers les strates de la mémoire personnelle, il permet l'accès à ce haut-lieu du sens qu'est la mémoire transpersonnelle. $A u$ risque de la pensée et de l'écriture, pour qu'advienne l'intensité, écris-tu dans la dédicace que tu m'adresses. C'est précisément dans cet esprit que je veux te lire. Comment s'y prendre pour que se fasse la lumière des interférences d'une écriture et d'une lecture? Existe-t-il une méthode? J'ai toujours su que la lumière mentale se fait comme se fait le miel. D'un butin «approprié» par l'abeille. J'ai lu et médité ton livre. J'en ferai une relecture d'abeille. De ta pensée, je m'approprierai ce qui en ce moment parle à la mienne. Le miel, ça s'élabore dans la ruche, du dialogue de l'abeille avec ce qu'elle a pris et compris des fleurs qu'elle a visitées pendant la journée.

Tu écris dès la première page:la subjectivité au féminin est une appropriation au sens d' une conquête. La réalité patriarcale exige d' une femme qu' elle passe par l' inavouable ou par l' effraction pour signifier sa raison. Plus loin: la nécessité de penser la raison appropriée - celle qui est adéquate et celle dont on 
s'empare - relève d' une expérience et d' un constat liés à un parcours d'écriture. La raison, donc, en tant que butin, qui correspond à une faim très précise et dont on éprouve qu'il est vital de s'emparer pour exister, pour trouver sa place au soleil dans la civilisation patriarcale; butin qu'on garde sur l'estomac comme un empêchement d'être - un surmoi paralysant - tant qu'on n'est pas capable de se l'assimiler, tant que le travail alchimique de l'écriture n'a pas réussi à se l'approprier, à s'en faire matière au verbe de soi. Ainsi es-tu amenée d reconnaître un désir de raison qui expose une part d' irrationalité. La raison du père comme objet du désir irraisonné, du désir fou de la fille. Ce que tu en dis m'amène, moi, à reconnaître que l'objet incestueux de ma plus folle passion fut effectivement ce qui me manquait le plus: la raison d'un père que je rêvais idéal, cette valeur virile en quoi la civilisation reconnaît le plus grand des biens: la Valeur en soi, celui qui n'en possède pas la maîtrise étant du côté de ce qui doit être dominé. Que la raison du Père puisse être objet suprême du désir de la fille, voilà qui déjà tient de l' inavouable.

D'entrée de jeu, ton livre - à travers l'auto-analyse — ouvre le procès de la civilisation patriarcale. Tout porte à croire que l'écriture des femmes l'instruira, car c'est la franchise du sujet féminin qu'elle gagne à l'analyse de son rapport à la raison. La psychanalyse qui fait du rapport à la folie son objet ne peut jamais que réaffirmer implicitement, comme allant de soi, l'exclusivité référentielle de la raison du père, modèle hors duquel il n'y a point d'ordre donc point de salut individuel ou collectif. Il faut reprendre a la psychanalyse le champ verbal de la connaissance psychique. Il faudra te savoir gré d'avoir osé l'écrire aussi impérativement. Cette décolonisation de la connaissance de soi sera le premier pas hors de l'Histoire patriarcale. Mais peut-être fallait-il que la psychanalyse, à l'instar de la science, devienne un piège pour que ce «pas au-delà» soit imaginable.

L'écriture qui advient lorsque la raison est dévastée par une mémoire blessée pèse ses mots. Car l'écriture telle que je la conçois est une expérience globale qui participe tout à la fois de l'être, du temps et de l'espace. Écriture où peut se déceler l'émergence de nouvelles formes de la subjectivité. Une telle écriture chercheuse d'issue suppose qu'ait été surmontée la colère, que la turbulence intérieure ait trouvé le point d'application de l'énorme énergie qu'elle brasse, que la pensée soit devenue une invincible volonté de comprendre, le lieu de cette fidélité à soi-même qui consiste à ne se laisser fragmenter qu'au nom d'une intégrité toujours à refaire en avant de soi, plus subtilement articulée, plus connaissante des tensions, des liens d'interaction et de transaction qu'entretiennent ses multiples lieux.

Écrire la souffrance, c'est davantage écrire la figure du guet-apens. L'énergie noire est une énergie piégée et lucide. Tu parles de la souffrance avec une intensité qui trahit l'ascèse à laquelle elle se doit. Il y a un seuil d'acuité de la souffrance du dominé où, ayant surmonté la fascination de l'issue fatale - meurtre ou suicide -, elle cesse de s'exécrer, moins pour compatir à elle-même que pour se rendre justice, et se percevoir comme bénéfice brut d'une «santé du malheur» qui prit son cours avec notre chute dans l'Histoire patriarcale. La souffrance 
accumulée dans la mémoire du dominé est une intarissable mine d'or mental. La souffrance des femmes vient souvent d' un malaise qui la dépasse. Je veux parler d' une souffrance très ancienne, celle de sa mère peut-être, celle d' une mémoire des femmes. L'énergie noire, que nul oubli ne peut anéantir, s'est capitalisée dans la mémoire des femmes. La fortune de souffrance dont nous sommes légataires en cette fin du deuxième millénaire a atteint ce seuil d'excédence où se produit un saut qualitatif. Dès que la souffrance ne peut plus se souffrir, elle s'interroge. La fin de l'Histoire commence avec l'interrogation du pire, dès que la mémoire de la douleur se découvre matière à lucidité, matière à conscience de conscience. De question en question, la souffrance qui alimente et meut la pensée fouilleuse remonte à la figuration du guet-apens, autrement dit au principe de raison duelle qui fonde tout savoir et tout pouvoir. Excluant le tiers, le principe de domination positivise, phallicise, divinise la raison du sujet dominant tandis qu'elle négativise, hystérise celle du sujet dominé. L'une marquée du sceau de Dieu, l'autre de celui du diable. L'Histoire est un piège divisionnaire où sont tombés dominants et dominés, hommes et femmes. Seule est capable de nous en sortir la souffrance des dominés dans la mesure où, s'excédant, elle se charge d'écrire la part non dite de l'Histoire, dont elle est la mémoire. Ton livre est riche de cette intelligence résistante à la fatalité de l'Histoire. Te lire et te relire, c'est à chaque ligne et entre les lignes trouver dans ton expérience de l'entre raison et déraison de quoi s'éclairer soi-même en tant que tiers, en tant que mitoyen.

Ce que tu désignes, dès l'introduction, par réalité patriarcale, ce qui nous est condition historique, c'est donc la dictature du sujet masculin. Cette dictature ne se perpétue que si la raison du Père se textualise pour pouvoir se transmettre de père en fils, et si ce texte qui constitue le «corps de la Loi» fait référence immuable, s'il est cela qu'il faut respecter, à quoi il faut être conforme pour avoir droit de cité. De ce processus de tradition du corps de la Loi, le sujet féminin est écarté, étant «impropre» à cette fonction. La mère, qui transmet la langue du père, est une bonne mère si elle fait aveuglément corps avec le corps de la Loi. Une mère qui s'écrit s'exclut. Faisant corps textuel avec elle-même, solidaire de la déraison, elle commet le plus sacrilège des adultères. Elle se met hors la Loi. L'exclusion devient sa condition réelle au regard de la tradition. Si on ne la lynche plus, si on ne la montre plus du doigt, la réprobation, surtout muette, l'encercle et l'isole. Une mère qui s'écrit épouse sa «folie», installe sa concubine - la révolution - dans la maison de la tradition. Elle trahit la confiance du Père. Tu as su cela très tôt: l'écriture, telle que je la conçois, est une expérience globale qui participe tout à la fois de l'être, du temps et de l'espace. Tu savais qu'un tel engagement dans l'écriture, et la maternité - telle qu'elle comble le désir du Père - étaient incompatibles. Ton texte intitulé «La vieille petite fille» est d'une lucidité exemplaire: Mon père $m^{\prime}$ 'a dit un jour que rien ne lui ferait plus plaisir si ce n'est que j'aie un enfant. Je n'ai jamais répondu d̀ une telle demande. Et je me suis sentie devenir au fur et d̀ mesure des années, dans un mouvement spiralé, une vieille petite fille. Pour moi, la venue tardive à l'écriture, à la conscience d'exister, a fait se résoudre le probleme tout autrement. J'ai Écrit dans mon demier livre: $J$ 'avais eu quatre enfants. Je sus très vite que je n'en ferais plus parce que ma fécondité m'était irrémédiablement montée d la tête. 
Enfants, petits-enfants... Il a fallu et il faut encore trouver des compromis, comme si le quotidien battait entre la loi et la folie, entre la famille, le travail rémunéré et l'écriture. Celle qui - d la limite de l' impudeur - s'autographie au jour le jour commence à pouvoir assumer sa culpabilité à l'égard de la tradition, le jour où envers et contre tout chantage, elle se reconnaît soi-même responsable de la postérité de la révolution, des enfants du rêve; à partir du jour où, à ses yeux, son existence n'a pas de plus grande utilité que de donner corps de texte - espace et temps du livre à la raison contraire. Entre la culpabilité à l'égard du passé et la culpabilité à l'égard du futur, il faut choisir. Nous sommes de celles qui, non sans douloureuses oscillations, ont décisivement préféré la première. Toi, vieille petite fille, et moi, grand-mère indigne, sommes à présent logées à la commune enseigne de celles qui entrent en écriture pour faire arriver ce qui $n$ 'existe pas encore et qui pourrait bien être le secret de la communauté.

La souveraineté de la raison du sujet masculin s'étant fondée sur le musellement de la déraison attribuée au sujet féminin, on pourrait dire que le silence du sujet féminin fut acheté - au nom de l'Ordre - contre une place plus ou moins enviable dans la Maison patriarcale (c'est ce qui s'appelle pour une souris, donner sa langue au chat). Ce silence est ainsi devenu propriété foncière de la raison masculine. Au nom de la Loi, elle y a droit. Si une femme rompt le contrat, si elle reprend sa «parole donnée», elle perd sa place en même temps que la protection du Maître. Elle est «renvoyée». Il faut entendre ici le mot «renvoi» au sens que lui donnait Rimbaud: Quand sera brisé l' infini servage de la femme, quand elle vivra pour elle et par elle, l' homme - jusqu' ici abominable - lui ayant donné son renvoi, elle sera poète, elle aussi! La femme trouvera de l'inconnu! Cette parole confisquée, il arrive un moment de l'Histoire où le sujet féminin veut à tout prix se la réapproprier. Elle ne le peut qu'en s'appropriant la raison elle-même. Ainsi la parole gelée depuis la nuit des temps dans les sous-sols de la raison geôlière, refait-elle irruption, non sans déstabiliser irréversiblement les structures de l'empire patriarcal. À l'issue de cette catastrophe, raison et déraison ne seront plus jamais ce qu'elles furent. S'étant éprouvées l'une l'autre, elles se découvriront fécondées l'une par l'autre, générant à leur corps défendant, des formes inédites de parole métisse, ces nouvelles formes de la subjectivité dont tu nous entretiens dans la troisième partie du livre.

L'écriture du délire exige qu' on croie en sa propre raison. La raison ordonne le délire, sans quoi il n'y a pas d'écriture possible. Ta réflexion sur les conditions du délire des écrivains masculins à propos des femmes met en lumière un fait mal aperçu jusqu'ici: c'est que l'objet du délire, s'il devient lui-même sujet pensant la condition du sujet délirant, devient «impropre» à la consommation. Un pur objet peut être fantasmé, un objet-sujet ne le peut puisqu'il s'approprie le sujet délirant, puisqu'il se fait de la raison délirante un objet d'analyse. En d'autres termes, la femme qui s'écrit, la démuselée, devient impropre à la fonction de muse; nous avons toutes fait la cuisante expérience. La complexification du rapport sujet-objet du désir est aujourd'hui telle qu'il est urgent pour la santé de la pensée d'en prendre acte. 
Le sujet masculin n'a pu fonder sa croyance en sa propre raison qu'en achetant le silence du sujet féminin, en achetant le droit de le fantasmer, de s'en inspirer, de le faire sien; en se l'appropriant, aux deux sens du terme. En achetant le droit au délire créateur qui seul dénoue la peur, l'êcrivain masculin délire impunément, voire même «hygiéniquement», sans attenter, dis-tu d̀ son unité, ou encore d̀ sa capacité d'émettre au sujet de la société une opinion fondée. Ce droit de fantasmer le sujet féminin, entre les limites extrêmes de la sanctification et de l'abjection, est, à bien y regarder, la sauvegarde même de l'Ordre patriarcal. Il n'y a qu' un seul corps de fantasmes pour l' un et l'autre sexe: le corps féminin. Ce corps de fantasmes, une femme peut en jouer à défaut d'en jouir, s'en faire un moyen de chantage, ou bien vouloir changer la langue. Entre autres, donner un contenu symbolique au mot «femme» et non plus à «l'éternel féminin». Entreprise - ô combien solitaire - de rachat du sujet féminin par l'écriture des femmes.

Une recherche sur le double sens de la propriété s'amorce dans ton livre qu'il faudra pousser en son dernier retranchement. Faire dire à ce mot-là ce qu'il n'a jamais dit. Il semble donc que ce qui permet au sujet de s'affirmer verbalement, c'est d'intérioriser, de s'approprier mentalement l'objet de son désir. En ce senslà, on peut dire que c'est l'avoir qui donne la parole à l'être, contrairement à ce qu'on a voulu nous faire croire. Si tu appartiens, tu n'es pas je, tu es l'autre, tu es dans l'aliénation. Se réappropriant son propre corps, s'appropriant la raison masculine qui l'a réduit à n'être qu'un corps de fantasmes, le sujet féminin retrouve la parole, mais parole, faut-il le dire, atrophiée par un long arrêt de croissance, par une longue absence à soi-même, une longue inhibition de sa propre jouissance. Pour retrouver la santé créatrice, le sujet n'a d'autre chance, d'autre urgence - envers et contre toute condamnation - d'avoir recours à l'inconditionnel amour de soi, à tous les moyens d'autoréanimation, d'autoréhabilitation. À partir du moment où une femme croit en elle, en sa valeur propre (à quel prix!), elle «voit» ce que l'incroyance en soi lui interdisait de voir. Les choses profondes lui deviennent transparentes, visibles et dicibles. Par exemple, elle découvre - non sans quelque malice bien légitime - que la raison virile appropriée et cultivée par la mentalité féminine a le pouvoir de réveiller la part de «folie» (part non dite, part maudite) qui sommeille au fond de la mentalité masculine. Lorsque la souris a repris sa langue au chat, elle est à lui ce qu'il est à elle. Dominant et dominé logés à la même enseigne mitoyenne, entre raison et déraison. La femme sera la première à réaliser que la vie a changé, que dans la relation des sexes la désinhibition de sa subjectivité a fait succéder le principe de réciprocité au principe d'identité, le principe de raison dialogique au principe de raison logique. Elle sera la première à comprendre que la virilité d'une femme ne s'identifie pas plus à la virilité d'un homme que la féminité d'un homme à celle d'une femme; que si les sexes sont théoriquement deux, ils sont réellement quatre, à partir de quoi leur différenciation se fait de plus en plus fine. À elle «d'être en avant», d'initier les enfants du siècle qui vient aux nouvelles formes de la subjectivité, à la pratique du «grand jeu» relationnel qui sera celui de la fratrie postpatriarcale. À elle de s'écrire, non plus pensant d̀ qui ne la lit pas, mais pensant à qui ne la lit pas encore, à sa postérité réelle. 
Reste l'importante question que tu poses dans la lettre intitulée «Femmes et pensée», dont je me reconnais la destinataire: Existe-t-il un délire créateur pour une écrivaine? Autrement dit, la mémoire culturelle permet-elle à l'écrivaine de maintenir une distance entre l'émotion et la raison? Nous en avions longuement parlé lors de mon dernier séjour à Montréal. Cette question est parmi celles que je ne me suis jamais posées avec une telle netteté. Elle m'avait d'autant plus vivement atteinte que venait de s'écrire, en parenthèses à mon dernier livre, un grand délire métamorphique intitulé «le désastre», déclenché par l'expérience intérieure d'avoir brisé la statue du Commandeur, autrement dit d'avoir fait exploser l'homme de pierre qui nous tient lieu de surmoi patriarcal. Depuis, il m'est arrivé souvent de me reposer ta question, de me l'approprier pour tenter d'y répondre. Sans aucun doute, Ariane, l'écrivaine, avait brisé la statue au terme d'un long et minutieux processus d'appropriation de la raison dominante. Et manifestement, c'était à cette condition qu'elle se sortirait elle-même de son enfermement dans la mémoire labyrinthique. Cette issue s'opéra à la faveur d'un outrage délirant de la fatalité mythologique qui voue le féminin à être fantasmé par le masculin; Ariane, Eurydice, Jocaste, Marguerite, Lilith et d'autres y prenaient ensemble les rennes de leur destin, bouleversaient allègrement l'Ordre mythologique fondateur de notre civilisation.

Entre raison et déraison, il n'y a qu' un pas, écris-tu. Ce pas avait été franchi par la raison dominée, résolue à reconquérir sa propriété foncière. L'explosion du surmoi gardien de l'étanchéité entre raison et déraison avait dû se produire d'un éclair d'évidence, d'une reconnaissance fulgurante de leur commune origine, reconnaissance qui pulvérisait tout fondement, toute légitimité d'une domination, d'une colonisation de l'une par l'autre. La question qui se pose ici maintenant concerne le «vide de Loi» laissé dans le psychisme par la liquidation plus ou moins brutale du surmoi patriarcal. La question de l'an-archie. C'est bien là le problème crucial de toute révolution individuelle et collective. Quelles structures pour une société postpatriarcale? La réponse qu'apporte mon expérience est celleci: à la logique symbolique du Commandeur succède la dialogique d'Ariane et de Don Juan (l'interlocuteur d'Ariane dans le «désastre»), tous deux orphelins de la Loi, se rencontrant sur les lieux de l'apocalypse. C'est là même, dans le vide d'éthique patriarcale, que se produit la genèse d'une éthique de la fraternité, de la réciprocité, de la solidarité; que naissent de nouvelles formes d'intersubjectivité où ordre et désordre, raison et déraison coexistent dynamiquement, créativement.

J'ai récemment compris qu'il arrive un moment crucial de l'expérience de soi où il faut choisir entre l'intégrité de la statue du Père et celle qu'entretient la présence à soi, entre la destruction des Tables de la Loi et l'autodestruction. Où c'est la statue qui se brise est évitée la désintégration de l'être réel. Dès que ces choses-là se divulguent, qui pourrait encore se résigner à périr «au nom de la Loi»? Moïse lui-même n'avait-il pas déjà brisé les premières Tables de la Loi?

Combien je te sais gré, également, d'avoir écrit: $J$ 'aurais envie de dire sérieusement ceci: depuis que les femmes écrivent au féminin, certaines d' entre elles ont détruit les représentations de la folie littéraire, elles sont entrées, sans 
devenir folles, dans les problématiques d Artaud et de Gauvreau, pour ne nommer que ces deux écrivains. Grâce d̀ eux, sans doute et d̀ cause d' elles en tout premier lieu, elles ont pu déconstruire certains schémas de langage, prendre des risques et ne pas se perdre dans certaines expériences liées aux limites de l'écriture. C'est dire que la société peut attendre des femmes qu'elles trouvent le passage et qu'elles passent là où l'écriture des hommes ne s'est jamais aventurée sans que se perde irréversiblement leur raison. Lorsque raison et déraison font alliance, envers et contre tout interdit, en vue de faire passer le fil de l'écriture par la petite porte du fond de la nuit, c'est signe d'une sagesse à venir. Signe que la chouette est de retour (René Char).

Il faudra que nous reprenions notre dialogue sur quelques points précis, dont je pressens qu'il pourrait être révélateur des différences réelles entre l'expérience de la mémoire profonde - de la mémoire religieuse, au sens étymologique, athéologique du terme - telle qu'elle est vécue par une écrivaine américaine et telle qu'elle l'est par une européenne. Nous connaissons bien les affinités qu'il peut y avoir entre une québécoise et une wallonne, il faudrait également partager la conscience des lieux de leur différence; en quelque sorte faire parler en français la mémoire transatlantique. Revenir aussi sur l'épreuve de maturation qu'est le passage mental de la dualité à la quadrature, sur la différence entre poétique et mystique, etc... Faire, en somme, un travail d'atelier sur les nouvelles formes de la subjectivité. La lecture féconde que je viens de faire de ton livre pourrait nous permettre, je crois, de faire un saut considérable dans notre recherche. Jusqu'ici - et je pèse mes mots -, je n'ai pas lu d'essai féminin qui atteigne à cette sensibilité lucide, qui m'instruise aussi spécifiquement des problèmes inhérents à l'écriture des femmes. Nul doute qu'il en aide plus d'une - et j'en jurerais, plus d'un - à se sortir d'une impasse, à faire quelques pas décisifs sur son propre chemin. 